\title{
Variación de Absorción en ladrillos de perlita cruda
}

\section{Variation of Absorption in raw perlite bricks}

Presentación: 07/10/2020

\section{Doctorando:}

\section{Lucas Ramiro Burgos}

Facultad de Ingeniería - Universidad Nacional de Salta, Argentina

Centro de Investigación, Desarrollo y Transferencia de Materiales y Calidad CINTEMAC - Universidad Tecnológica Nacional - Facultad Regional Córdoba, Argentina

lucasburgos@conicet.gov.ar

\section{Director/es: \\ Ángel Oshiro}

\section{Co-director/es:}

\section{María Inés Sastre}

\section{Resumen}

Los ladrillos de perlita cruda presentan una opción para la utilización de desechos de perlita cruda que se generan en la zona de la puna en la provincia de Salta, ya que presentan resistencias a la compresión superiores a 5 Mpa a los 90 días de su fabricación y pueden ser utilizados para la fabricación de viviendas sociales. Pese a esto, posee un alto porcentaje de absorción respecto a su peso, alrededor del 25\%. Esto es debido a que su fabricación se realiza con compactación manual sin requerimiento de energía eléctrica. Más allá de que dicho mampuesto está destinado a zonas de clima desértico, se analizarán distintos tipos de recubrimientos hidrófugos, siguiendo la Norma IRAM 12588, para comparar la variabilidad de la absorción frente al costo y calidad del material hidrófugo.

Palabras clave: Mampuesto, Perlita cruda, Sustentabilidad, Absorción.

\begin{abstract}
Raw perlite bricks present an option for the use of raw perlite waste generated in the puna area in the province of Salta, since they present compressive strengths greater than 5 Mpa 90 days after their manufacture and they can be used for the manufacture of social housing. Despite this, it has a high absorption percentage with respect to its weight, around $20 \%$. This is due to the fact that its manufacture is carried out with manual compaction without requiring electrical energy. Beyond the fact that this masonry is intended for desert climate areas, different types of waterproof coatings will be analyzed, following the IRAM 12588 Standard, to compare the variability of absorption versus the cost and quality of the waterproof material.
\end{abstract}

Keywords: Masonry, Raw perlite, Sustainability, Absorption. 


\section{Introducción}

La actividad minera es uno de los rubros con mayor desempeño al oeste de la provincia de Salta. Esto a su vez beneficia económicamente a la zona, pero en contraparte genera desechos mineros, los cuales son controlados periódicamente por las autoridades de Minería de la Provincia de Salta. A través de ellos se accedió a los desechos de perlita cruda, los cuales fueron utilizados para la fabricación de mampuestos en la Facultad de Ingeniería de la Universidad Nacional de Salta. Se fabricaron bloques y ladrillos, estos últimos presentaron resultados favorables para su utilización en viviendas sociales. No solo una resistencia a la compresión mayor a los 5Mpa luego de 90 días de maduración, sino que además presentan un coeficiente de conductividad térmica similar a los ladrillos macizos cerámicos que se ofrecen en el mercado. (Burgos et al., 2019)

Más allá de las características positivas nombradas, los ladrillos de perlita cruda presentan un alto porcentaje de absorción comparada con los estándares que hay en el mercado. (Guerrero Gómez et al., 2017)

El alto porcentaje de absorción se debe a la manera en la que es fabricado el ladrillo. Para ello, inicialmente se mezcal en seco las proporciones de cemento, cal hidratada y perlita cruda. Luego, tanto la mezcla como la compactación en un equipo CINVA RAM se realizan de manera manual buscando no trabajar con energía eléctrica, ya que la zona donde está destinada existen sectores donde no cuentan con suministro eléctrico. (Burgos et al., 2019)

\section{Desarrollo}

Para el desarrollo de este trabajo se sigue la Norma IRAM 12588. Particularmente el método de ensayo para la determinación de la capacidad de absorción de agua por inmersión en agua fría.

Se secan las muestras hasta masa constante y luego se pintan con los productos impermeabilizantes, siguiendo las recomendaciones de los fabricantes. Luego se sumergen 24 horas, se toman las masas y con ello se calculan los porcentajes de absorción.

Para ello se seleccionan tres marcas distintas de impermeabilizante, de distintos precios y calidad.

\section{Resultados}

Al momento en que se presenta este trabajo, no se han podido conocer los resultados, ya que no se puede acceder al laboratorio como consecuencia de la pandemia.

El objetivo es conocer si realmente es importante la variación de absorción para introducir un costo adicional a unos mampuestos destinado a vivienda sociales.

\section{Referencias}

Burgos, L. R., Oshiro, Á., \& Sastre, M. I. (2019). Influencia de Finos de Perlitas para la Fabricación de Ladrillos y Bloques. Uso en Viviendas Sociales. AJEA. https://doi.org/10.33414/ajea.4.369.2019

Guerrero Gómez, G., Espinel Blanco, E., \& Sánchez Acevedo, H. G. (2017). Análisis de temperaturas durante la cocción de ladrillos macizos y sus propiedades finales. Revista Tecnura. https://doi.org/10.14483/udistrital.jour.tecnura.2017.1.a09 